PROCEEDINGS OF THE

AMERICAN MATHEMATICAL SOCIETY

Volume 135, Number 2, February 2007, Pages 397-403

S 0002-9939(06)08741-7

Article electronically published on August 2, 2006

\title{
LOCAL CAUCHY-RIEMANN EMBEDDABILITY OF REAL HYPERBOLOIDS INTO SPHERES
}

\author{
JONG-WON OH \\ (Communicated by Mei-Chi Shaw)
}

\begin{abstract}
In this paper, we study the local Cauchy-Riemann embeddability of strictly pseudoconvex real hyperboloids $M^{2 n+1}$ into spheres. By solving a $\mathrm{CR}$ analogue of the Gauss equation, we prove that $M^{2 n+1}$ is CR-embeddable into spheres with a $\mathrm{CR}$ co-dimension $<n-1$ if and only if it is spherical.
\end{abstract}

\section{Introduction AND STATEMENT OF RESULT}

The purpose of this paper is to investigate the obstructions for strictly pseudoconvex Cauchy-Riemann (CR) manifolds $M^{2 n+1}$ of hypersurface type to be locally embedded by CR mapping into higher dimensional spheres $S^{2 N+1}$. We will only consider the smooth category in this paper.

We are motivated by the study of proper holomorphic embeddability into balls. Let $D$ be a smoothly bounded strictly pseudoconvex domain in $\mathbb{C}^{n+1}$. Every $D$ can be properly holomorphically embedded into balls $\mathbb{B}^{N+1}$ for some $N \geq n$ so that embedding extends to boundary continuously or Hölder continuously (see [5], 9], [10]), but not every $D$ can be embedded so that embedding extends to boundary smoothly (see [4], [5]).

The authors of [4] and [5] derived the above by showing that the set of germs of CR-embeddable $M^{2 n+1}:=\partial D$ is of the first category in the space of germs of real-analytic strictly pseudoconvex hypersurfaces. This observation leads to a study of obstructions to local CR embeddability into spheres. CR co-dimension between $M^{2 n+1}$ and a sphere is kept finite, otherwise there is no obstruction to CR embeddability according to 8 . Thus we are led to:

Problem 1. Characterize strictly pseudoconvex CR manifolds $M^{2 n+1}$ which are locally CR-embeddable into spheres with finite CR co-dimensions. State all the obstructions to the embeddability of such manifolds in terms of the Chern-Moser invariants.

We shall investigate simple models in this paper. A smooth strictly pseudoconvex CR manifold of hypersurface type which is flat in the sense of Chern-Moser [1] is locally equivalent to the standard sphere

$$
|Z|^{2}+|w|^{2}=1, \quad(Z, w) \in \mathbb{C}^{n} \times \mathbb{C} .
$$

Received by the editors August 25, 2005.

2000 Mathematics Subject Classification. Primary 32V30; Secondary 32V20.

Key words and phrases. CR embeddability, real hyperboloid, sphere, the Gauss equation.

The author was supported by BK21-Yonsei University.

(C)2006 American Mathematical Society Reverts to public domain 28 years from publication 
By the local holomorphic change of coordinates $Z \rightarrow Z / w$ and $w \rightarrow 1 / w$, it is equivalent to

$$
|Z|^{2}-|w|^{2}=-1, \quad(Z, w) \in \mathbb{C}^{n} \times \mathbb{C},
$$

a standard real hyperboloid. This is biholomorphically equivalent to a sphere minus some proper analytic subvariety, hence it is strictly pseudoconvex everywhere. Both are obviously CR-embeddable into another sphere of any positive CR co-dimension.

Now we deform each by simple quadratic perturbation. Perturb the standard sphere into real ellipsoids $r(Z, w, \bar{Z}, \bar{w})=0$, where

$$
r=\sum_{\alpha=1}^{n}\left|z^{\alpha}\right|^{2}+\sum_{\alpha=1}^{n} a_{\alpha}\left(\left(z^{\alpha}\right)^{2}+\left(z^{\bar{\alpha}}\right)^{2}\right)+|w|^{2}+a\left(w^{2}+\bar{w}^{2}\right)-1
$$

for $Z=\left(z^{1}, \ldots, z^{n}\right)$ and $-1 / 2<a_{\alpha}, a<1 / 2$. Also perturb the standard real hyperboloid into real hyperboloids $r(Z, w, \bar{Z}, \bar{w})=0$, where

$$
r=\sum_{\alpha=1}^{n}\left|z^{\alpha}\right|^{2}+\sum_{\alpha=1}^{n} a_{\alpha}\left(\left(z^{\alpha}\right)^{2}+\left(z^{\bar{\alpha}}\right)^{2}\right)-|w|^{2}-a\left(w^{2}+\bar{w}^{2}\right)+1,
$$

which are strictly pseudoconvex near the points of $Z=0$. We raise the question: when are these two CR manifolds locally CR-embeddable into spheres with a positive CR co-dimension? Surprisingly, these two examples show different CR embeddability. For the first case, we have:

Theorem 1.1 (13, 3]). All general real ellipsoids of the class (1.3) are locally CR-embeddable into the sphere with $C R$ co-dimension 1.

Proof. A holomorphic mapping $G(Z, w)=\left(Z, w, i / 2-i \sum_{\alpha=1}^{n} a_{\alpha}\left(z^{\alpha}\right)^{2}-i a w^{2}\right)$ from $\mathbb{C}^{n+1}$ to $\mathbb{C}^{n+2}$ embeds the real ellipsoid (1.3) into Heisenberg group $\mathbb{H}^{2 n+3}=$ $\left\{2 \operatorname{Im} z^{n+2}=\sum_{\alpha=1}^{n+1}\left|z^{\alpha}\right|^{2}\right\}$ which is locally CR-equivalent to a sphere in $\mathbb{C}^{n+2}$.

On the other hand, for the second case, we prove

Theorem 1.2. A germ at $Z=0$ of the real hyperboloid of the class (1.4) is CRembeddable into spheres with a positive $C R$ co-dimension $<n-1$ if and only if

$$
a_{1}=a_{2}=\cdots=a_{n}=a=0 .
$$

First we compute the CR curvature of our real hyperboloid by means of pseudohermitian geometry. Then we derive a CR analogue of the Gauss equation by means of a pseudohermitian embedding system: the CR-Gauss equation was used for rigidity problems in Webster [12] and in Ebenfelt, Huang and Zaitsev [2]. We solve the CR-Gauss equation by means of Huang's lemma and conclude Theorem 1.2. Finally we discuss our result and propose related problems.

\section{CR AND PSEUdohermitian CURVATURES}

In this section we shall compute the pseudohermitian curvature of our real hyperboloids (1.4) and translate it into Chern-Moser's CR curvature. Throughout this paper we adopt the summation convention and let the Greek indices $\alpha, \beta, \ldots$ run over the set $1,2, \ldots, n$; lower indices $a, b, \ldots$ run over $n+1, \ldots, N$, and upper indices $A, B, \ldots$ run over $1,2, \ldots, N$. 
Let $M^{2 n+1}$ be a real hyperboloid $r(Z, w, \bar{Z}, \bar{w})=0$, where

$$
r=-\sum_{\alpha=1}^{n}\left|z^{\alpha}\right|^{2}-\sum_{\alpha=1}^{n} a_{\alpha}\left(\left(z^{\alpha}\right)^{2}+\left(z^{\bar{\alpha}}\right)^{2}\right)+|w|^{2}+a\left(w^{2}+\bar{w}^{2}\right)-1,
$$

with $Z=\left(z^{1}, \ldots, z^{n}\right),-1 / 2<a_{\alpha}, a<1 / 2$ for $\alpha=1, \ldots, n$. We have altered the sign of each term in (1.4) to make the Levi form positive definite at $Z=0$. Choose a standard contact form $\theta=i \partial r$. In [1], S. Webster showed that there exist coframe $\left(\theta, \theta^{\alpha}, \theta^{\bar{\alpha}}\right)$, unique 1-form $\omega_{\beta}^{\alpha}$, pseudohermitian torsion $A_{\alpha \gamma}$ and curvature $R_{\beta \bar{\alpha} \nu \bar{\mu}}$ satisfying

$$
\begin{aligned}
d \theta & =i g_{\alpha \bar{\beta}} \theta^{\alpha} \wedge \theta^{\bar{\beta}}, \\
d \theta^{\alpha} & =\theta^{\beta} \wedge \omega_{\beta}^{\alpha}+A_{\bar{\mu}}^{\alpha} \theta \wedge \theta^{\bar{\mu}} \quad \text { with } \quad d g_{\beta \bar{\alpha}}=\omega_{\beta \bar{\alpha}}+\omega_{\bar{\alpha} \beta}, \\
d \omega_{\beta}^{\alpha} & =\omega_{\beta}^{\gamma} \wedge \omega_{\gamma}^{\alpha}+R_{\beta \nu \bar{\mu}}^{\alpha} \theta^{\nu} \wedge \theta^{\bar{\mu}} \quad \bmod \theta, \theta^{\beta} \wedge \theta^{\nu}, \theta^{\bar{\alpha}} \wedge \theta^{\bar{\mu}} .
\end{aligned}
$$

The Levi form $g_{\alpha \bar{\beta}}$ is positive definite at $Z=0$, and hence $M^{2 n+1}$ is strict pseudoconvex on a neighborhood of $Z=0$. We make use of $g_{\alpha \bar{\beta}}$ and its inverse $g^{\beta \bar{\alpha}}$ to raise and lower indices. For our real hyperboloid (2.1) with $\theta^{\alpha} \equiv d z^{\alpha} \bmod \theta$,

$$
\left.R_{\beta \bar{\alpha} \nu \bar{\mu}}\right|_{0}=4 a_{\beta \nu} a_{\bar{\alpha} \bar{\mu}} Q-\left(g_{\nu \bar{\alpha}} g_{\beta \bar{\mu}}+g_{\beta \bar{\alpha}} g_{\nu \bar{\mu}}\right) Q
$$

where $a_{\alpha \beta}:=\delta_{\alpha \beta} a_{\alpha}, Q:=1 /(2 a w+\bar{w})(2 a \bar{w}+w), g_{\alpha \bar{\beta}}=\delta_{\alpha \bar{\beta}}+O\left(|Z|^{2}\right)$ and " $\left.\right|_{0}$ " denotes evaluation at points of $Z=0$. Furthermore, if $a_{1}=\cdots=a_{n}=0$, then the curvature on a neighborhood of $Z=0$ is

$$
R_{\beta \bar{\alpha} \nu \bar{\mu}}=-\frac{\left(g_{\nu \bar{\alpha}} g_{\beta \bar{\mu}}+g_{\beta \bar{\alpha}} g_{\nu \bar{\mu}}\right) Q}{1-Q|Z|^{2}}+\frac{4 a^{2} z^{\bar{\beta}} z^{\alpha} z^{\bar{\nu}} z^{\mu} Q^{3}}{1-Q|Z|^{2}}
$$

according to [14], where $0<Q \leq(1-2|a|)^{-1} /\left(1+|Z|^{2}\right)$.

Our coframe $\left(\theta, \theta^{\alpha}, \theta^{\bar{\alpha}}\right)$ is a section of pseudohermitian bundle $P \rightarrow M^{2 n+1}$. If we set $\phi=0$, then $\left(\theta, \theta^{\alpha}, \theta^{\bar{\alpha}}, \phi\right)$ is also a section of $Y \rightarrow M^{2 n+1}$ in Chern-Moser [1]. Thus we can compute CR curvatures from pseudohermitian torsion and curvature by, for instance,

$$
S_{\beta \bar{\alpha} \nu \bar{\mu}}=R_{\beta \bar{\alpha} \nu \bar{\mu}}-\frac{R_{\beta \bar{\alpha}} g_{\nu \bar{\mu}}+R_{\nu \bar{\alpha}} g_{\beta \bar{\mu}}+g_{\beta \bar{\alpha}} R_{\nu \bar{\mu}}+g_{\nu \bar{\alpha}} R_{\beta \bar{\mu}}}{n+2}+\frac{R\left(g_{\beta \bar{\alpha}} g_{\nu \bar{\mu}}+g_{\nu \bar{\alpha}} g_{\beta \bar{\mu}}\right)}{(n+1)(n+2)}
$$

with $R_{\beta \bar{\alpha}}=R_{\beta \bar{\alpha} \gamma}^{\gamma}$ and $R=R_{\nu}^{\nu}$. See [11] and [2]. Hence CR curvatures for (2.5) and (2.6) are, respectively,

$$
\left.S_{\beta \bar{\alpha} \nu \bar{\mu}}\right|_{0} \equiv 4 a_{\beta \nu} a_{\bar{\alpha} \bar{\mu}} Q \bmod (g)
$$

and

$$
S_{\beta \bar{\alpha} \nu \bar{\mu}} \equiv \frac{4 a^{2} z^{\bar{\beta}} z^{\alpha} z^{\bar{\nu}} z^{\mu} Q^{3}}{1-Q|Z|^{2}} \bmod (g),
$$

where "三 $\bmod (g) "$ denote the equality modulo $g_{\beta \bar{\alpha}} T_{\nu \bar{\mu}}+g_{\beta \bar{\mu}} T_{\nu \bar{\alpha}}+g_{\nu \bar{\mu}} T_{\beta \bar{\alpha}}+$ $g_{\nu \bar{\alpha}} T_{\beta \bar{\mu}}$ for some Hermitian tensor $T_{\beta \bar{\alpha}}$.

\section{Embedding system And the Gauss Equation}

In this section we shall derive a system for local CR embedding into spheres. We obtain the pseudohermitian Gauss equation and translate it into CR analogue. 
Suppose a real hyperboloid $M^{2 n+1}$ is locally CR-embedded in a sphere $S^{2 N+1}$. We can adapt a coframe on $S^{2 N+1}$ to an embedding, say $f$, by means of

Lemma $3.1([2])$. Let $M^{2 n+1}$ and $\hat{M}^{2 N+1}, N \geq n$, be $C^{\infty}$ smooth strictly pseudoconvex $C R$ manifolds of hypersurface type and let $f: M^{2 n+1} \rightarrow \hat{M}^{2 N+1}$ be a local smooth $C R$ embedding. For any coframe $\left(\theta, \theta^{\alpha}, \theta^{\bar{\alpha}}\right)$ satisfying (2.2) on $M^{2 n+1}$ with Levi form $g_{\alpha \bar{\beta}}$, there exist a contact form $\hat{\theta}$ and coframe $\left(\hat{\theta}, \hat{\theta}^{A}, \hat{\theta}^{\bar{A}}\right)$ on $\hat{M}^{2 N+1}$ with Levi form $\hat{g}_{A \bar{B}}$ such that (a) $f^{*}\left(\hat{\theta}, \hat{\theta}^{\alpha}, \hat{\theta}^{a}\right)=\left(\theta, \theta^{\alpha}, 0\right)$, (b) $d \hat{\theta}=i \hat{g}_{A \bar{B}} \hat{\theta}^{A} \wedge \hat{\theta}^{\bar{B}}$ and (c) $\hat{g}_{\alpha \bar{\beta}}=g_{\alpha \bar{\beta}}, \quad \hat{g}_{\alpha \bar{b}}=0, \quad \hat{g}_{a \bar{b}}=\delta_{a \bar{b}}$ on $f\left(M^{2 n+1}\right)$.

Denote coframe, connection, torsion and curvature on $\hat{M}^{2 N+1}$ with hat notation. Let $\Gamma:=\left\{(x, f(x)): x \in M^{2 n+1}\right\} \subset M^{2 n+1} \times \hat{M}^{2 N+1}$, and we shall understand every differential form as pulled back to $\Gamma$ and drop pull-back notations. The adapted coframe on $\hat{M}^{2 N+1}$ in Lemma 3.1 satisfies

$$
\begin{aligned}
& \hat{\theta}-\theta=0, \\
& \hat{\theta}^{\alpha}-\theta^{\alpha}=0, \\
& \hat{\theta}^{a}=0 .
\end{aligned}
$$

Differentiate (3.3) and apply (3.1), (3.2), (3.3) and (2.2) to obtain $0=d \hat{\theta}^{a}=\theta^{\beta}$ $\wedge \hat{\omega}_{\beta}{ }^{a}$ modulo $\hat{\theta} \wedge \hat{\theta}^{\bar{\mu}}$. By Cartan's lemma,

$$
\hat{\omega}_{\beta}^{a}=\omega_{\beta \nu}^{a} \theta^{\nu} \quad \text { and } \quad \hat{\omega}_{\bar{\beta} a}=\hat{\omega}_{\bar{\beta}}^{\bar{A}} \hat{g}_{a \bar{A}}=\hat{\omega}_{\bar{\beta}}^{\bar{b}} \hat{g}_{a \bar{b}}=\omega_{\bar{\beta} \bar{\mu}}^{\bar{b}} \hat{g}_{a \bar{b}} \theta^{\bar{\mu}}=\omega_{\bar{\beta} a \bar{\mu}} \theta^{\bar{\mu}}
$$

where $\omega_{\beta \nu}^{a}=\omega_{\nu \beta}^{a}$ shall be called the second fundamental form for the embedding.

Differentiate (3.2) and substitute (2.3), (2.4), (3.1), (3.2) and (3.3) to obtain 0 $=d\left(\hat{\theta}^{\alpha}-\theta^{\alpha}\right)=\theta^{\beta} \wedge\left(\hat{\omega}_{\beta}^{\alpha}-\omega_{\beta}^{\alpha}\right)$ modulo $\theta \wedge \theta^{\bar{\mu}}$. By Cartan's lemma,

$$
\hat{\omega}_{\beta}^{\alpha}-\omega_{\beta}^{\alpha}=C_{\beta \gamma}^{\alpha} \theta^{\gamma} \text { for } C_{\beta \gamma}^{\alpha}=C_{\gamma \beta}^{\alpha} \text {. }
$$

Combine (2.3), Lemma 3.1(c), (3.5) and its conjugate to obtain $0=d \hat{g}_{\beta \bar{\alpha}}-d g_{\beta \bar{\alpha}}$ $=\left(\hat{\omega}_{\beta}{ }^{A} \hat{g}_{A \bar{\alpha}}-\omega_{\beta}^{\gamma} g_{\gamma \bar{\alpha}}\right)+\left(\hat{\omega}_{\bar{\alpha}}^{\bar{A}} \hat{g}_{\bar{A} \beta}-\omega_{\bar{\alpha}}^{\bar{\gamma}} g_{\bar{\gamma} \beta}\right)=C_{\beta \bar{\alpha} \nu} \theta^{\nu}+C_{\bar{\alpha} \beta \bar{\nu}} \theta^{\bar{\nu}}$. Hence $C_{\beta \bar{\alpha} \nu}=0$ and (3.5) implies

$$
\hat{\omega}_{\beta}^{\alpha}-\omega_{\beta}^{\alpha}=0 \text {. }
$$

Differentiate (3.6) and substitute (2.4), (3.1), (3.2), (3.3) and (3.6) to obtain 0 $=d\left(\hat{\omega}_{\beta}^{\alpha}-\omega_{\beta}^{\alpha}\right)=\hat{\omega}_{\beta}^{a} \wedge \hat{\omega}_{a}^{\alpha}+\left(\hat{R}_{\beta \nu \bar{\mu}}^{\alpha}-R_{\beta \nu \bar{\mu}}^{\alpha}\right) \theta^{\nu} \wedge \theta^{\bar{\mu}}$ modulo $\theta, \theta^{\beta} \wedge \theta^{\nu}$ and $\theta^{\bar{\alpha}}$ $\wedge \theta^{\bar{\mu}}$. Lower the index $\alpha$ using Lemma 3.1(c) and substitute $\hat{\omega}_{a \bar{\alpha}}=-\hat{\omega}_{\bar{\alpha} a}+d \hat{g}_{a \bar{\alpha}}$ $=-\hat{\omega}_{\bar{\alpha} a}$ and (3.4) to obtain

$$
-\omega_{\beta}^{a}{ }_{\nu} \omega_{\bar{\alpha} a \bar{\mu}}+\hat{R}_{\beta \bar{\alpha} \nu \bar{\mu}}-R_{\beta \bar{\alpha} \nu \bar{\mu}}=0 .
$$

Substitute (3.7) into (2.7) and obtain the CR analogue of the Gauss equation (see [2] and [12]). If $\hat{M}^{2 N+1}$ is a sphere $S^{2 N+1}$, the CR curvature tensor $\hat{S}_{B \bar{A} C \bar{D}}$ vanishes and the Gauss equation reads

$$
\begin{aligned}
& S_{\beta \bar{\alpha} \nu \bar{\mu}}=-\omega_{\beta}^{a}{ }_{\nu} \omega_{\bar{\alpha} a \bar{\mu}}+\frac{1}{n+2}\left(\omega_{\gamma \beta}^{a} \omega_{a \bar{\alpha}}^{\gamma} g_{\nu \bar{\mu}}+\omega_{\gamma \nu}^{a} \omega_{a \bar{\alpha}}^{\gamma} g_{\beta \bar{\mu}}\right. \\
& \left.+g_{\beta \bar{\alpha}} \omega_{\gamma \nu}^{a} \omega_{a \bar{\mu}}^{\gamma}+g_{\nu \bar{\alpha}} \omega_{\gamma \beta}^{a} \omega_{a \bar{\mu}}^{\gamma}\right)-\frac{\omega_{\gamma}^{a} \sigma^{\gamma} \omega_{a}^{\sigma}}{(n+1)(n+2)}\left(g_{\beta \bar{\alpha}} g_{\nu \bar{\mu}}+g_{\nu \bar{\alpha}} g_{\beta \bar{\mu}}\right) .
\end{aligned}
$$




\section{CR EMBEDDABILITY OF REAL HYPERBOLOIDS INTO SPHERES}

In this section, we prove our main

Theorem 1.2. A germ at $Z=0$ of the real hyperboloid $r(Z, w, \bar{Z}, \bar{w})=0$, where

$$
r=\sum_{\alpha=1}^{n}\left|z^{\alpha}\right|^{2}+\sum_{\alpha=1}^{n} a_{\alpha}\left(\left(z^{\alpha}\right)^{2}+\left(z^{\bar{\alpha}}\right)^{2}\right)-|w|^{2}-a\left(w^{2}+\bar{w}^{2}\right)+1
$$

for $-1 / 2<a_{\alpha}, a<1 / 2$, is CR-embeddable into the spheres of positive $C R$ codimension $<n-1$ if and only if

$$
a_{1}=a_{2}=\cdots=a_{n}=a=0 .
$$

Proof. The "if" part is trivial. We prove the "only if" part. Let $M^{2 n+1}$ be the real hyperboloid in $\mathbb{C}^{n} \times \mathbb{C}$ given above and let $S^{2 N+1}$ be a sphere in $\mathbb{C}^{N} \times \mathbb{C}$ with the positive $\mathrm{CR}$ co-dimension $N-n<n-1$. We alter the sign of each term in (4.1) as in (2.1) to make the Levi form $g_{\beta \bar{\alpha}}$ positive definite on the points of $M^{2 n+1}$ with $Z=0$. Then Chern's CR curvature tensor of $M^{2 n+1}$ in (2.8) evaluated on $Z=0$ is

$$
\left.S_{\beta \bar{\alpha} \nu \bar{\mu}}\right|_{0} \equiv 4 Q a_{\beta \nu} a_{\bar{\alpha} \bar{\mu}} \bmod (g),
$$

and the Gauss equation (3.8) at the same point on $Z=0$ for a local smooth CR embedding $f: M^{2 n+1} \rightarrow \hat{M}^{2 N+1}$ is

$$
\left.S_{\beta \bar{\alpha} \nu \bar{\mu}}\right|_{0} \equiv-\sum_{c=n+1}^{N} \omega_{\beta}^{c} \omega_{\bar{\alpha} \bar{\mu}}^{\bar{c}} \bmod (g),
$$

where the Levi form $g_{\beta \bar{\alpha}}$ at $Z=0$ is $\delta_{\beta \bar{\alpha}}$. Combine (4.2) and (4.3) to obtain the system of equations in the second fundamental forms $\omega_{\beta}^{c}{ }_{\nu}$. We now derive necessary conditions on curvatures of $M^{2 n+1}$ or $a_{\beta \nu}$ in order for this system to have a solution. To solve the system, we convert it into a polynomial identity. Fix a reference point with $Z=0$ on the real hyperboloid (2.1). Define polynomials

$$
S(\zeta, \bar{\zeta}):=S_{\beta \bar{\alpha} \nu \bar{\mu}} \zeta^{\beta} \zeta^{\bar{\alpha}} \zeta^{\nu} \zeta^{\bar{\mu}}, \quad \omega^{c}(\zeta):=\omega_{\beta}^{c}{ }_{\nu} \zeta^{\beta} \zeta^{\nu}, \quad A(\zeta):=a_{\beta \nu} \zeta^{\beta} \zeta^{v},
$$

for $\zeta=\left(\zeta^{1}, \ldots, \zeta^{n}\right) \in \mathbb{C}^{n}$. Observe that each polynomial uniquely determines each tensor involved. Compute $S(\zeta, \bar{\zeta})$ from (4.2) and (4.3) and identify one with the other. Noting that any term $\equiv 0 \bmod (g)$ turns into the multiple of $|\zeta|^{2}$, we get

$$
\sum_{c=n+1}^{N}\left|\omega^{c}(\zeta)\right|^{2}+4 Q|A(\zeta)|^{2}=|\zeta|^{2} G(\zeta, \bar{\zeta})
$$

for some quadratic function $G$. To solve this, we apply the following.

Lemma 4.2 (Huang [6]). Let $\left\{\psi^{j}\right\}_{j=1}^{k}$ and $\left\{\chi_{j}\right\}_{j=1}^{k}$ be holomorphic functions in $z \in \mathbb{C}^{n}$ near the origin. Assume that $\psi_{j}(0)=\chi_{j}(0)=0$. Let $H(z, \bar{z})$ be a realanalytic function for $z$ sufficiently close to the origin such that

$$
\sum_{j=1}^{k} \psi_{j}(z) \overline{\chi_{j}(z)}=|z|^{2} H(z, \bar{z}) .
$$

Then when $k<n, H(z, \bar{z}) \equiv 0$. 
The number of summands in the left-hand side of (4.4) is $N-n+1<n$ by the assumption on CR co-dimension. The lemma asserts that the left-hand side of (4.4) vanishes. But $Q$ is positive and every summand is nonnegative. Hence

$$
\omega^{c}(\zeta)=A(\zeta)=0
$$

This in turn implies by the symmetry of the tensors that

$$
\omega_{\beta \nu}^{c}=0, \quad a_{\beta \nu}=0 .
$$

In particular, $a_{\alpha}=a_{\alpha \alpha}=0$ for $\alpha=1, \ldots, n$. Fix a new reference point with $Z \neq 0$ sufficiently close to 0 in $\mathbb{C}^{n}$. The $\mathrm{CR}$ curvature at the point is

$$
S_{\beta \bar{\alpha} \nu \bar{\mu}}=\frac{4 a^{2} z^{\bar{\beta}} z^{\alpha} z^{\bar{\nu}} z^{\mu} Q^{3}}{1-Q|Z|^{2}} \bmod (g)
$$

from (2.9), and the Gauss equation turns into polynomial identity

$$
\sum_{c=n+1}^{N}\left|\omega^{c}(\zeta)\right|^{2}+\frac{4 a^{2} Q^{3}\left|\langle\zeta, Z\rangle^{2}\right|^{2}}{1-Q|Z|^{2}}=|\zeta|^{2} \tilde{G}(\zeta, \bar{\zeta})
$$

where $\langle$,$\rangle is a standard Hermitian inner product and \tilde{G}$ is some quadratic function. The number of summands in the left-hand side is $N-n+1<n$ and Huang's lemma applies again: the left-hand side vanishes. But every summand is nonnegative. Hence $\omega^{c}(\zeta)=0$ and

$$
4 a^{2} Q^{3}\left|\langle\zeta, Z\rangle^{2}\right|^{2} /\left(1-Q|Z|^{2}\right)=0 \quad \text { for every } \zeta \in \mathbb{C}^{n} .
$$

Since $Q>0$ and $Z \neq 0$, we have $a=0$, and the theorem follows.

\section{Discussion of Results AND Further PROBlems}

In this section, we shall review our results and propose further problems. A flat strictly pseudoconvex CR manifold $M^{2 n+1}$ of hypersurface type can be locally embedded in $\mathbb{C}^{n} \times \mathbb{C}$ as a standard sphere (1.1) or a standard real hyperboloid (1.2). Local quadratic deformations of $M^{2 n+1}$ in each embedding lead into general real ellipsoids (1.3) or general strictly pseudoconvex real hyperboloids (1.4). Let us call these ellipsoidal deformation and hyperboloidal deformation, respectively. These are generically no longer CR flat.

Put $M^{2 n+1}$ in another sphere of positive $\mathrm{CR}$ co-dimension by a suitable $\mathrm{CR}$ embedding. Can we make ellipsoidal or hyperboloidal deformation of $M^{2 n+1}$ in a higher-dimensional sphere? S. Webster observed that ellipsoidal deformation meets no obstruction in the ambient sphere, but our Theorem 1.2 shows that hyperboloidal deformation is obstructed by the CR structure of the ambient sphere and hence is not realizable when $\mathrm{CR}$ co-dimension is less than the CR dimension of $M^{2 n+1}$ minus one. Now it is natural to ask

Problem 2. If the CR co-dimension is large enough, then can a strictly pseudoconvex real hyperboloid (1.4) be locally CR-embedded into the spheres?

In the proof of Theorem 1.2. we made use of Huang's lemma and assumed that we had a suitably small $\mathrm{CR}$ co-dimension. If the $\mathrm{CR}$ co-dimension is the same as the CR dimension of $M^{2 n+1}$ minus 1, then Huang's lemma is not applicable. X. Huang and S. Ji [7] dealt with such a borderline CR co-dimension in their classification of 
CR embedding from $\partial \mathbf{B}^{n}$ into $\partial \mathbf{B}^{2 n-1}$; i.e., between the boundaries of unit balls in complex spaces. We propose

Problem 3. If the CR co-dimension is the same as the CR dimension of $M^{2 n+1}$ minus 1, then what is the CR embeddability of hyperboloidal deformation of $M^{2 n+1}$ in the sphere of a positive co-dimension?

\section{ACKNOWLEDGMENT}

The author sincerely extends his gratitude to Professor Ebenfelt for his guidance and fruitful advice on this work during the author's visit to University of California, San Diego, in 2002.

\section{REFERENCES}

[1] Chern, S. S. and Moser, J. K., Real hypersurfaces in complex manifolds, Acta Math. 133 (1974), 219-271. MR0425155 (54:13112)

[2] Ebenfelt, P., Huang, X. and Zaitsev, D., Rigidity of CR-immersions into spheres, Comm. Anal. Geom. 12 (2004), no. 3, 631-670. MR2128606 (2006c:53037)

[3] Ebenfelt, P., Huang, X. and Zaitsev, D., The equivalence problem and rigidity for hypersurfaces embedded into hyperquadrics, Amer. J. Math. 127 (2005), no. 1, 169-191. MR 2115664 (2006c:32047)

[4] Faran, J., The nonembeddability of real hypersurfaces in spheres, Proc. Amer. Math. Soc. 103 (1988), 902-904. MR0947678(89i:32037)

[5] Forstnerič, F., Embedding strictly pseudoconvex domains into balls, Trans. Amer. Math. Soc. 295 (1986), 347-368. MR0831203 (87k:32052)

[6] Huang, X., On a linearity problem for proper holomorphic maps between balls in complex spaces of different dimensions, J. Differential Geom. 51 (1999), 13-33. MR.1703603 (2000e:32020)

[7] Huang, X. and Ji, S., Mapping $\mathbf{B}^{n}$ into $\mathbf{B}^{2 n-1}$, Invent. Math. 145 (2001), 219-250. MR:1872546 (2002i:32013)

[8] Lempert, L., Imbedding Cauchy-Riemann manifolds into a sphere, International J. of Math. 1 (1990), 91-108. MR.1044662 (91c:32013)

[9] Løw, E., Embeddings and proper holomorphic maps of strictly pseudoconvex domains into polydiscs and balls, Math. Z. 190 (1985), 401-410. MR0806898 (87b:32047)

[10] Stensønes, B., Proper maps which are Lipschitz $\alpha$ up to the boundary, J. Geom. Anal. 6 (1996), no. 2, 317-339. MR1469126 (98g:32040)

[11] Webster, S. M., Pseudohermitian structures on a real hypersurface, J. Differential Geom. 13 (1978), 25-41. MR0520599 (80e:32015)

[12] Webster, S. M., The rigidity of $C$ - $R$ hypersurfaces in a sphere, Indiana Univ. Math. J. 28 (1979), 405-416. MR0529673 (80d:32022)

[13] Webster, S. M., Segre polar correspondence and double valued reflection for general ellipsoids, Analysis and geometry in several complex variables (Katata, 1997), Trends Math., Birkhäuser, Boston, MA (1999), 273-288. MR1699856 (2000k:32033)

[14] Webster, S. M., A remark on the Chern-Moser tensor, Houston J. Math. 28 (2002), no. 2, 433-435. MR 1898199 (2003d:32047)

Department of Mathematics, Yonsei University, Seoul 120-749, Korea

E-mail address: jwoh@math.snu.ac.kr

Current address: Department of Mathematics, Seoul National University, San 56-1, Sillimdong, Gwanak-gu, Seoul 151-742, Korea 